\title{
Identification of Immune Infiltration, Differentially Expressed Genes, and Signaling Pathways in Fanconi Anemia Based on Bioinformatics Analysis
}

\section{Biyu Shen}

Shanghai Children's Medical Center

\section{Songsong Shi}

Shanghai Children's Medical Center

\section{Haoyang Chen}

Shanghai Children's Medical Center

Yi Lu (D luyi@scmc.com.cn )

Shanghai Children's Medical Center

Hengmei Cui

Shanghai Children's Medical Center

\section{Yunyun Li}

Shanghai Children's Medical Center

\section{Dongliang Yang}

Guangzhou Medical University

\section{Research Article}

Keywords: Fanconi anemia, immune infiltration, signaling pathways, differentially expressed genes, bioinformatics

Posted Date: January 3rd, 2022

DOI: https://doi.org/10.21203/rs.3.rs-1215771/v1

License: (a) (1) This work is licensed under a Creative Commons Attribution 4.0 International License. Read Full License 


\section{Abstract}

Background and Objective: Fanconi anemia (FA) patients have a reduced ability to form blood cells, accompanied by multiple congenital malformations, mental retardation, solid tumors, and other symptoms. However, the molecular mechanism that causes FA is unclear, and few studies have addressed the regulatory mechanism of immune infiltration in FA. Here, we aimed to identify differentially expressed genes (DEGs), pathways, and immune infiltration involved in FA using integrated bioinformatics analysis and molecular mechanisms.

Methods: The GEO gene chip database was searched for FA low density bone marrow tissue, and the content and proportion of 22 types of immune cells in the FA group and the normal group were analyzed using CIBERSORT. Gene Ontology (GO) and Kyoto Encyclopedia of Genes and Genomes (KEGG) analysis of FA differentially expressed genes (DEGs) using $\mathrm{R}$ language and related package programs was also performed.

Results: The expression levels of T cells regulatory (Tregs), M2 macrophages, T cells CD8, dendritic cells resting, and T cells CD4 naïve in FA were higher than in the normal group. Furthermore, the expression levels of naïve B cells, monocytes, and resting mast cells in FA were lower than in the normal group. GO analysis of FA differential genes showed that "neutrophil degranulation," "neutrophil activation," and "neutrophil activation involved in immune response," were most frequently enriched among biological processes, with "specific granule," "tertiary granule," "tertiary granule lumen" among cellular components, and "carbohydrate binding" among molecular functions. For the KEGG analysis, "Asthma" was most often enriched.

Conclusion: This study obtained useful data related to immune infiltration, DEGs, and gene pathways of FA, and provides new evidence for immunotherapy and clinical assessment of FA patients. These results are potentially a useful reference for subsequent related scientific research.

\section{Background}

Fanconi anemia (FA) is a rare, autosomal recessive inherited disease of the circulatory system[1]. It is characterized by premature aging, serious failure of bone marrow development, and a high susceptibility to various tumors[2, 3]; among them, the probability of FA patients developing acute myeloid leukemia is 700-fold that of other populations[4]. The incidence of FA is about 1-5 per million, and the probability of being a heterozygous carrier is 1 in 300[5]. FA is usually more common in childhood, with an average age at diagnosis of 7 years [6]; only around $9 \%$ of patients are diagnosed in adulthood[7]. Some FA patients are born to consanguineous families [8]. The clinical manifestations of FA patients are obviously heterogeneous, and multiple systems are usually involved. One or more lines of cytopenia may occur at a young age, accompanied by multiple congenital malformations, mental development disorders, and solid tumors at an early stage. Typical patients are easily diagnosed, but about a third of FA patients do not have the above-mentioned malformations, and only present with aplastic anemia or solid tumors. For 
these reasons, it is easy to misdiagnose and therefore miss the best time for treatment[9]. Without treatment, FA evolves into progressive bone marrow failure (BMF), and is most often fatal before the end of the second decade of life[10]. Early detection and treatment of patients with FA should therefore be emphasized. The chromosome break test is currently the gold standard for diagnosing FA [11]. However, some patients do produce false negative or false positive results[12]. The current understanding of FA has developed from the chromosomal level to the gene level and it has been shown that germline inactivation of any FA pathway gene leads to this disease [13]. Genetic testing is essential for the diagnosis of FA, along with the identification of specific genetic mutations that cause the disease.

FA is the most common type of congenital hematopoietic failure [14, 15], and hematopoietic stem cell transplantation is the only long-term remission for patients. However, although hematopoietic stem cell transplantation can solve the problem of bone marrow failure, it cannot prevent the occurrence of tumors [16]. After transplantation, it is still possible to progress to gastrointestinal, head and neck, esophagus, vulvar, and anal cancers[17]; therefore, there is an urgent need to find new treatments. Gene therapy can avoid the main adverse effects of hematopoietic stem cell transplantation, such as endocrine diseases and solid malignancies; this is a promising path [18]. In recent years, the progress of molecular genetic research has contributed to the comprehensive study of FA, and, as the molecular mechanism of FA has become clearer, gene therapy has become possible[19-21]. However, owing to the limitations of current disease models, the pathogenesis of FA is still not fully understood[22]. It is also not clear how the bone marrow microenvironment in FA affects the pathogenesis of bone marrow failure[23]. Therefore, further exploration of the bone marrow microenvironment of FA is important for optimizing treatment plans and improving the survival rate of patients with FA. At present, the treatment of FA combines a pretreatment of chemotherapy followed by the transplantation of hematopoietic stem cells with cell removal [24, 25]; gene therapy is still at the clinical research stage. It is therefore of interest to study the potential molecular mechanism of FA, and find more reliable molecular markers and more effective diagnostic techniques to detect its occurrence and evaluate prognoses. Furthermore, the genetic and molecular mechanisms of developmental problems need to be further clarified. This research aimed to discover the pattern of immune cell infiltration and to distinguish differentially expressed genes (DEGs) in patients with FA in order to provide a basis for future diagnosis and treatment.

By searching the data sets related to FA in the Gene Expression Omnibus (GEO) database, we found that GSE16334(https://www.ncbi.nlm.nih.gov/gds/?term=GSE163 34) met the research conditions (21 FA samples and 11 normal samples). We entered them into the CIBERSORT database (https://cibersort.stanford.edu/) [26] to analyze the composition of immune cells. We then used R software (version 3.6.1) to process the downloaded files, and utilized histograms, principal component maps, heat maps, and other visualization methods to highlight the differences in immune cells between the FA group and normal group. By using co-expressing heat maps, violin maps, etc., we accurately described the differences in immune cell infiltration between the FA group and normal group.

For FA patients and healthy individuals, comprehensive analysis of the differential gene data in GEO data can be implemented, to provide a basis for the immune infiltration mechanism and intervention measures 
related to immune regulation of FA. Owing to the limited number of samples and differences in selection, not all selected DEGs, related pathways, and immune infiltrations can be directly used as biomarkers.

\section{Results}

\section{Immune Cell Infiltration Distribution and Significantly Different Expressions in FA Tissues and Normal} Tissues

We downloaded 11 normal samples and 21 FA samples, and entered them into the CIBERSORT database to compute the content of immune cells; the results were drawn as a histogram (Figure 1A). We then drew the results using a principal component diagram, to determine whether the content of immune cells in the FA group and the normal group were significantly different. Finally, we drew a heat map and analyzed the expression of immune cells (Figure 1B). By drawing a violin chart, we discovered that the expression levels of T cells regulatory (Tregs), M2 macrophages, $T$ cells CD8, dendritic cells resting, and T cells CD4 naïve in FA were higher than in the normal group, and the expression levels of resting mast cells, naïve $B$ cells, and monocytes in FA were lower than in the normal group (Figure 1C).

\section{Co-expression Analysis between Immune Cells}

The co-expression analysis of immune cell expression levels received in this research showed that M2 macrophages had the strongest positive correlation with memory B cells, with a correlation coefficient of 0.74 , and eosinophils had a strong positive correlation with M1 macrophages, with a correlation coefficient of 0.72 . We also found that CD8 T cells, follicular helper T cells, and M1 macrophages were significantly positively correlated. CD8 T cells were strongly negatively correlated with monocytes and mast cells resting, with correlation coefficients of -0.75 and -0.71 , respectively (Figure 2 ).

\section{Principal Component Analysis (PCA)}

We conducted PCA to determine whether these immune cells could be differentiated between the FA group and the normal group. The dimensionality was reduced to PCA1 and PCA2 by PCA; the X-axis is marked as PCA1 and Y-axis as PCA2. Ellipses were then simulated of the normal group and the FA group, respectively. We employed the "GGplot2" software package for analysis, and found that the two ellipses did not cross (Figure 3), demonstrating that the 22 types of immune cells in the research clearly distinguished the FA group from the normal group.

\section{Screening of Immune-related Genes}

During analysis of immune-related genes with the visualized heatmap, we found that 16 genes were upregulated and 21 genes were down-regulated among individuals with FA (Figure 4).

\section{GO Enrichment Analysis and KEGG Pathway Analysis of DEGs}


GO and KEGG enrichment analyses of 37 DEGs were performed using the R software "clusterProfiler" package. The biological processes mainly involved neutrophil activation involved in immune response, neutrophil degranulation, and neutrophil activation. The cellular components mainly involved tertiary granule, specific granule, and tertiary granule lumen. The molecular functions mainly involved carbohydrate binding. Based on KEGG enrichment analysis, these genes were involved in the regulation of immune-related pathways, such as "Asthma" (Figure 5).

\section{Discussion}

FA impacts all organs of the body. It has profound effects far beyond the disease itself, early diagnosis and treatment may be delayed until bone marrow failure begins and prevent severe complications[27], therefore scholars have increased their research efforts regarding FA in recent years. Due to the development of second-generation sequencing, greater numbers of researchers have begun to use bioinformatics technology to study FA. Hou et al.[28] showed that HIST1H1D, HIST1H3A, PSME1, and THRAP3 may play important roles in the progression of FA to acute myeloid leukemia (AML) and that they may be used as markers for the early diagnosis of AML. At around the same time, Degan et al. [29] used bioinformatics to predict target genes for the differentially expressed miRNAs and to conduct pathway enrichment analysis. Concurrently, Bogliolo et al.[30]also developed a simplified and effective methodology of FA molecular diagnosis based on whole exome sequencing (WES) and functional research. Velimezi et al.[31]discovered that USP48 can control DNA repair and recommended it as a potential target for the treatment of FA. One year later, Hou et al. [32] showed that bioinformatics analyses could be used to identify biomarkers and pathways related to FA and AA. They found that some key DEPs, such as ENO1, ACTC1, ACTBL2, EEF1A1, PGK1, and CFL1, may play an important role in FA and show potential as serological markers for early diagnosis. Compared with previous research, this study applied bioinformatics technology to the analysis of FA immune cell infiltration to comprehensively identify markers that can better diagnose FA, but also analyzed immune-related genes and identified differentially expressed genes, in order to provide reference for future diagnostics and therapy.

In the current study, we analyzed the GSE16334 datasets related to FA, then determined immune cell infiltration in FA and the correlation between immune cells. Furthermore, we identified DEGs and analyzed the functional pathway enrichment of DEGs. We found that the expressions of M2 macrophages, Tregs, CD8 T cells, CD4 naïve T cells, and resting dendritic cells in the FA group were higher than those in the normal group. Meanwhile, the expressions of naïve B cells, resting mast cells, and monocytes in FA were lower than those in the normal group. These differences provide more evidence for our clinical diagnosis of FA; furthermore, some of the results predicted by the experiment have been confirmed by basic research. $B$ cells are essential for humoral based immunity and previous studies have shown the effect of FA deficiency in B cell function. The overall transcriptome analysis of naïve B cells by RNAseq shows that FA deficiency can relieve the gene network involved in immune function [33]. In FA patients regulatory $T$ cells, and naïve T cells were increased relative to the control group. Regarding naïve T cells, an additional explanation for the relative increase may come from the proliferation caused by steady-state lymphopenia. When lymphocyte counts decrease and cell cycle activation occurs, this is a compensatory 
mechanism and is related to the acquisition of a naïve phenotype[21] .Studies have shown that CD8 T cells are reduced or not significantly changed in FA; moreover, the results of this study showed that CD8 T cells were more abundant in the FA group than in the control group. It cannot be ruled out that the small sample size may have caused the CIBERSORT algorithm to be biased in calculating the number of immune cells[34, 35].

In the co-expression analysis between immune cells, correlations were compared between 22 cell types. The results showed that the positive correlation between M2 macrophages and memory B cells was the strongest, some scholars have reported the same results in tumors[36], and monocytes were strongly negatively correlated with resting mast cells, Some scholars got the same result in tumors[37], reflecting the synergy or antagonism between different immune cells in the progression of FA. The current basis for analysis of the correlation between different types of immune cells still lacks large-scale verification, this prediction has a certain degree of reference significance.

GO and KEGG enrichment analyses were used to describe the relationships among DEGs. GO functional annotation revealed that the hub genes were mainly involved in immune responses. Those genes closely related to immune regulation were mainly involved in biological process, cellular components, and molecular functions; there was also an intricate transduction process. KEGG enrichment analysis showed that hub genes were involved in the regulation of immune-related pathways, for instance the asthma signaling pathway. The correlation between the asthma signaling pathway and FA needs further confirmation in future studies.

The limitations of our research were as follows: (1) As no experiments were undertaken, our results cannot be validated; there is therefore a need for clinical molecular experiments to confirm the identified genes and pathways in FA. (2) The data used in this research came from publicly available databases, therefore we could not assess the quality of the data, which might have led to bias; (3) There were only a few bioinformatic tools used in this research; future research should include more bioinformatic tools and more FA datasets should be used to identify further KEGG pathways. (4) Finally, as Fanconi anemia is a rare disease, the sample size was relatively small, which will have affected gene expression.

\section{Conclusion}

In this study, a series of results were obtained through the in-depth analysis of chip data, which were used to predict the distribution of FA immune cells, infiltration characteristics, and to examine the interaction between FA relevant DEGs. These findings will contribute to a better understanding of the molecular mechanisms of FA. At the same time, this study proposed a new method for analyzing the relationship between immune cells and FA, which provides new evidence for the immunotherapy of FA patients. There is a need for further related molecular experiments to confirm the gene functions associated with FA.

\section{Materials And Methods}




\section{Data Download}

This study used the GEO database (https://www.ncbi.nlm.nih.gov/geo/) to look for relevant data of patients with FA and discovered that the GSE16334 data set met our study needs. It contained data for 21 FA samples and 11 healthy controls.

\section{Immune Infiltration Matrix}

CIBERSORT is widely used to evaluate relative cell content and dynamic regulation processes. The 22 immune cell types included M0 macrophages, M1 macrophages, M2 macrophages, follicular helper T cells, CD8 T cells, regulatory T cells (Tregs), gamma delta T cells, CD4 naïve T cells, CD4 memory activated T cells, CD4 memory resting T cells, naïve B cells, memory B cells, plasma cells, neutrophils, activated mast cells, monocytes, activated natural killer (NK) cells, resting NK cells, activated dendritic cells, eosinophils, resting dendritic cells, and resting mast cells. We downloaded 32 samples of the data set and computed the relevant immune cell content in CIBERSORT.

\section{Heat Maps Related to Immune Cells}

The pheatmap package was applied to analyze and draw a heat map of the relationship between immune cells and the expression of the sample visually.

\section{Correlation Heat Maps of Immune Cells}

The R language "corrplot" package was used to draw heat maps and analyze correlations of the immune cells expressed in the samples.

\section{Violin Diagram of Immune Cell Expression in Normal Tissues and FA Tissues}

The R language "vioplot" software package was used to analyze immune cells and draw a violin chart. Red indicates the FA group, and blue indicates the normal group.

\section{Principal Component Analysis (PCA) of Infiltrated Immune Cells}

PCA is a multiple regression analysis algorithm; we used it to assess the infiltration of 22 types of immune cells between the two different groups. Two circles were used to differentiate the FA group and the normal group, and a dot indicates a sample.

\section{Identification of Differentially Expressed Genes (DEGs)}

The operating instruction codes of the "limma" package in R software (https://bioconductor.org/biocLite.R) were applied to screen the DEGs between the FA group and normal group; this information was then utilized for further analysis. The volcano chart shows all DEGs that were upregulated and downregulated. Then, using the "pheatmap" package and limiting the project to 37 DEGs, hierarchical clustering was applied. Heat maps were used to display the results. 


\section{Functional and Pathway Enrichment Analysis of DEGs}

The R software "clusterProfiler" package(http://www.bioconductor.org/packages/relea se/bioc/html/clusterProfiler.html)was used to determine GO function and KEGG pathway enrichment analysis of the different genes. Hypergeometric distribution was used to analyze and compute the significance levels of these DEGs in each signaling pathway and to identify the significantly affected signaling pathways $(P<0.05)$.

\section{Abbreviations}

FA $\triangle$ Fanconi anemia $\triangle D E G s ~ \triangle D$ ifferentially Expressed Genes; GO :Gene Ontology; KEGG :Kyoto Encyclopedia of Genes and Genomes; BMF: bone marrow failure; GEO :Gene Expression Omnibus ; NK :natural killer ; PCA :Principal Component Analysis.

\section{Declarations}

\section{Acknowledgements}

Not applicable.

\section{Authors' contributions}

$\mathrm{BY}, \mathrm{SS}$, and $\mathrm{HC1+:}$ conception, design, and performance of the research and writing of the paper. $\mathrm{YL}^{*}$ : supervision of the research. $\mathrm{HC}$ and $\mathrm{YL}$ : provision of useful suggestions in methodology. $\mathrm{HC} 1+$ and DY: provision of suggestions in figure preparation. All authors read and approved the final version of the manuscript. All authors read and approved the final manuscript.

\section{Funding}

Not applicable.

\section{Availability of data and materials}

Not applicable.

\section{Ethics approval and consent to participate}

Not applicable.

\section{Consent for publication}

Not applicable.

\section{Competing interests}


The authors declare that they have no competing interests.

\section{References}

[1] Ahammad F, Rupa HS, Rashid AM and Rasul CH. Fanconi anemia. Bangladesh Medical Journal Khulna 2017;50 (1-2): 46-48. https://doi.org/10.3329/bmjk.v50i1-2.35844

[2] Deng S, Ye W, Zhang S, et al. Oral Tongue Cancer in a Patient with Fanconi Anemia: A Case Report and Literature Review. Cancer Manag Res. 2021;13:3145-3154. https://doi.org/10.2147/CMAR.S301582

[3] Guitton-Sert L, Gao Y, Masson JY. Animal models of Fanconi anemia: A developmental and therapeutic perspective on a multifaceted disease. Semin Cell Dev Biol. 2021;113:113-

131.https://doi.org/10.1016/j.semcdb.2020.11.010

[4] Nepal M, Che R, Zhang J, Ma C, Fei P. Fanconi Anemia Signaling and Cancer. Trends Cancer. 2017;3(12):840-856. https://doi.org/10.1016/j.trecan.2017.10.005

[5] Jacquemont C, Taniguchi T. The Fanconi anemia pathway and ubiquitin. BMC Biochem. 2007;8 Suppl 1(Suppl 1):S10. Published 2007 Nov 22. https://doi.org/10.1186/1471-2091-8-S1-S10

[6] Fiesco-Roa MO, Giri N, McReynolds LJ, Best AF, Alter BP. Genotype-phenotype associations in Fanconi anemia: A literature review. Blood Rev. 2019;37:100589. https://doi.org/10.1016/j.blre.2019.100589

[7] Alter BP. Diagnosis, genetics, and management of inherited bone marrow failure syndromes. Hematology Am Soc Hematol Educ Program. 2007;29-39.

https://doi.org/10.1182/asheducation-2007.1.29

[8] Balta G, Patiroglu T, Gumruk F. Fanconi Anemia and Ataxia Telangiectasia in Siblings who Inherited Unique Combinations of Novel FANCA and ATM Null Mutations. J Pediatr Hematol Oncol. 2019;41(3):243-246. https://doi.org/10.1097/MPH.0000000000001336

[9] Mundlos S and Horn D. Fanconi Anemia. In: editors. Limb Malformations. Springer; 2014. p. 193-194.

[10] Ben Haj Ali A, Messaoud O, Elouej S, et al. FANCA Gene Mutations in North African Fanconi Anemia Patients. Front Genet. 2021;12:610050. https://doi.org/10.3389/fgene.2021.610050

[11] Oostra AB, Nieuwint AW, Joenje $H$, de Winter JP. Diagnosis of fanconi anemia: chromosomal breakage analysis. Anemia. 2012;2012:238731. https://doi.org/10.1155/2012/238731

[12] Auerbach AD. Diagnosis of Fanconi anemia by diepoxybutane analysis. Curr Protoc Hum Genet. 2015;85:8.7.1-8.7.17. https://doi.org/10.1002/0471142905.hg0807s85

[13] Rodríguez A, Naveja JJ, Torres L, et al. WIP1 Contributes to the Adaptation of Fanconi Anemia Cells to DNA Damage as Determined by the Regulatory Network of the Fanconi Anemia and Checkpoint 
Recovery Pathways. Front Genet. 2019;10:411.https://doi.org/10.3389/fgene.2019.00411

[14] Nicoletti E, Rao G, Bueren JA, et al. Mosaicism in Fanconi anemia: concise review and evaluation of published cases with focus on clinical course of blood count normalization. Ann Hematol. 2020;99(5):913-924. https://doi.org/10.1007/s00277-020-03954-2

[15] Rodríguez A, Zhang K, Färkkilä A, et al. MYC Promotes Bone Marrow Stem Cell Dysfunction in Fanconi Anemia. Cell Stem Cell. 2021;28(1):33-47.e8. https://doi.org/10.1016/j.stem.2020.09.004

[16] Yabe M, Morio T, Tabuchi K, et al. Long-term outcome in patients with Fanconi anemia who received hematopoietic stem cell transplantation: a retrospective nationwide analysis. Int $\mathrm{J}$ Hematol. 2021;113(1):134-144. https://doi.org/10.1007/s12185-020-02991-x

[17] García MJ, Fernández V, Osorio A, et al. Analysis of FANCB and FANCN/PALB2 fanconi anemia genes in BRCA1/2-negative Spanish breast cancer families. Breast Cancer Res Treat. 2009;113(3):545551.https://doi.org/10.1007/s10549-008-9945-0

[18] Tolar J, Becker PS, Clapp DW, et al. Gene therapy for Fanconi anemia: one step closer to the clinic. Hum Gene Ther. 2012;23(2):141-144. https://doi.org/10.1089/hum.2011.237

[19] Shafqat S, Tariq E, Parnes AD, Dasouki MJ, Ahmed SO, Hashmi SK. Role of gene therapy in Fanconi anemia: A systematic and literature review with future directions. Hematol Oncol Stem Cell Ther. 2021;14(4):290-301. https://doi.org/10.1016/j.hemonc.2021.02.001

[20]Czechowicz A, Agarwal R, Sevilla J, Río P and Roncarolo MG. Gene Therapy for Fanconi Anemia, Complementation Group a: Updated Results from Ongoing Global Clinical Studies of RP-L102. Blood .2020; 136: 14-14. https://doi.org/ 10.1182/blood-2020-137106

[21] Czechowicz A , Rio P , Bueren J E , et al. Changing the Natural History of Fanconi Anemia Complementation Group- with Gene Therapy: Early Results of U.S. Phase I Study of Lentiviral-Mediated Ex-Vivo FANCA Gene Insertion in Human Stem and Progenitor Cells. Biology of Blood and Marrow Transplantation, 2020, 26(3) . https://doi.org/10.1016/ j.bbmt.2019.12.106

[22] Solanki A, Rajendran A, Mohan S, Raj R, Vundinti BR. Mitochondrial DNA variations and mitochondrial dysfunction in Fanconi anemia. PLoS One.

2020;15(1):e0227603.https://doi.org/10.1371/journal.pone.0227603

[23] Zhou Y, He Y, Xing W, et al. An abnormal bone marrow microenvironment contributes to hematopoietic dysfunction in Fanconi anemia. Haematologica. 2017;102(6):10171027. https://doi.org/10.3324/haematol.2016.158717

[24] Alhuraiji A, Alzahrani H, Al Mohareb F, et al. Hematopoietic Stem Cell Transplant in Adolescent and Young Adults With Fanconi Anemia Is Feasible With Acceptable Toxicity, With Those Surviving 100 Days 
Posttransplant Having Excellent Outcomes. Exp Clin Transplant. 2016;14(6):660664. https://doi.org/10.6002/ect.2015.0364

[25] Strocchio L, Pagliara D, Algeri M, et al. HLA-haploidentical TCRaß+/CD19+-depleted stem cell transplantation in children and young adults with Fanconi anemia. Blood Adv. 2021;5(5):1333-1339. https://doi.org/10.1182/bloodadvances.2020003707

[26] Zheng R, Wan C, Mei S, et al. Cistrome Data Browser: expanded datasets and new tools for gene regulatory analysis. Nucleic Acids Res. 2019;47(D1):D729-D735. https://doi.org/10.1093/nar/gky1094

[27] Bhandari J, Thada P K, Puckett Y. Fanconi anemia. StatPearls, 2021.

[28] Hou H, Li D, Gao J, et al. Proteomic profiling and bioinformatics analysis identify key regulators during the process from fanconi anemia to acute myeloid leukemia. Am J Transl Res. 2020;12(4):14151427. PMID: 32355551

[29] Degan P, Cappelli E, Longobardi M, et al. A Global MicroRNA Profile in Fanconi Anemia: A Pilot Study. Metab Syndr Relat Disord. 2019;17(1):53-59. https://doi.org/10.1089/met.2018.0085

[30] Bogliolo M, Pujol R, Aza-Carmona M, et al. Optimised molecular genetic diagnostics of Fanconi anaemia by whole exome sequencing and functional studies. J Med Genet. 2020;57(4):258268. https://doi.org/10.1136/jmedgenet-2019-106249

[31] Velimezi G, Robinson-Garcia L, Muñoz-Martínez F, et al. Map of synthetic rescue interactions for the Fanconi anemia DNA repair pathway identifies USP48. Nat Commun.

2018;9(1):2280. https://doi.org/10.1038/s41467-018-04649-z

[32] Hou H, Li D, Yao YH, et al. Proteomic analysis for identifying the differences in molecular profiling between fanconi anaemia and aplastic anaemia. Am J Transl Res. 2019;11(10):65226533. PMID: 31737203

[33] Sertorio M, Amarachintha S, Wilson A, Pang Q. Loss of Fancc Impairs Antibody-Secreting Cell Differentiation in Mice through Deregulating the Wnt Signaling Pathway. J Immunol. 2016;196(7):29862994. https://doi.org/10.4049/jimmunol.1501056

[34] Myers KC, Sauter S, Zhang X, et al. Impaired immune function in children and adults with Fanconi anemia. Pediatr Blood Cancer. 2017;64(11):10.1002/pbc.26599. https://doi.org/10.1002/pbc.26599

[35] Justo GA, Bitencourt MA, Pasquini R, et al. Immune status of Fanconi anemia patients: decrease in T CD8 and CD56dim CD16+ NK lymphocytes. Ann Hematol. 2014;93(5):761-

767. https://doi.org/10.1007/s00277-013-1953-4

[36] Wang Z, Wang Y, Peng M, Yi L. UBASH3B Is a Novel Prognostic Biomarker and Correlated With Immune Infiltrates in Prostate Cancer. Front Oncol. 
[37] Cao H, Quan S, Zhang L, Chen Y, Jiao G. BMPR2 expression level is correlated with low immune infiltration and predicts metastasis and poor survival in osteosarcoma. Oncol Lett. 2021;21(5):391. https://doi.org/10.3892/ol.2021.12652

\section{Figures}

\section{Figure 1}

Immune cell infiltration analysis. (A) The distribution of 22 types of immune cells between FA tissues and normal tissues. From right to left, the first 21 samples are FA tissues, the remaining samples are normal tissues. (B) A heatmap. Red indicates upregulation and green indicates downregulation. Explanation of "type": blue indicates normal tissue and red indicates FA tissue. (C) Violin diagram. Blue = normal group; red $=$ FA group . 


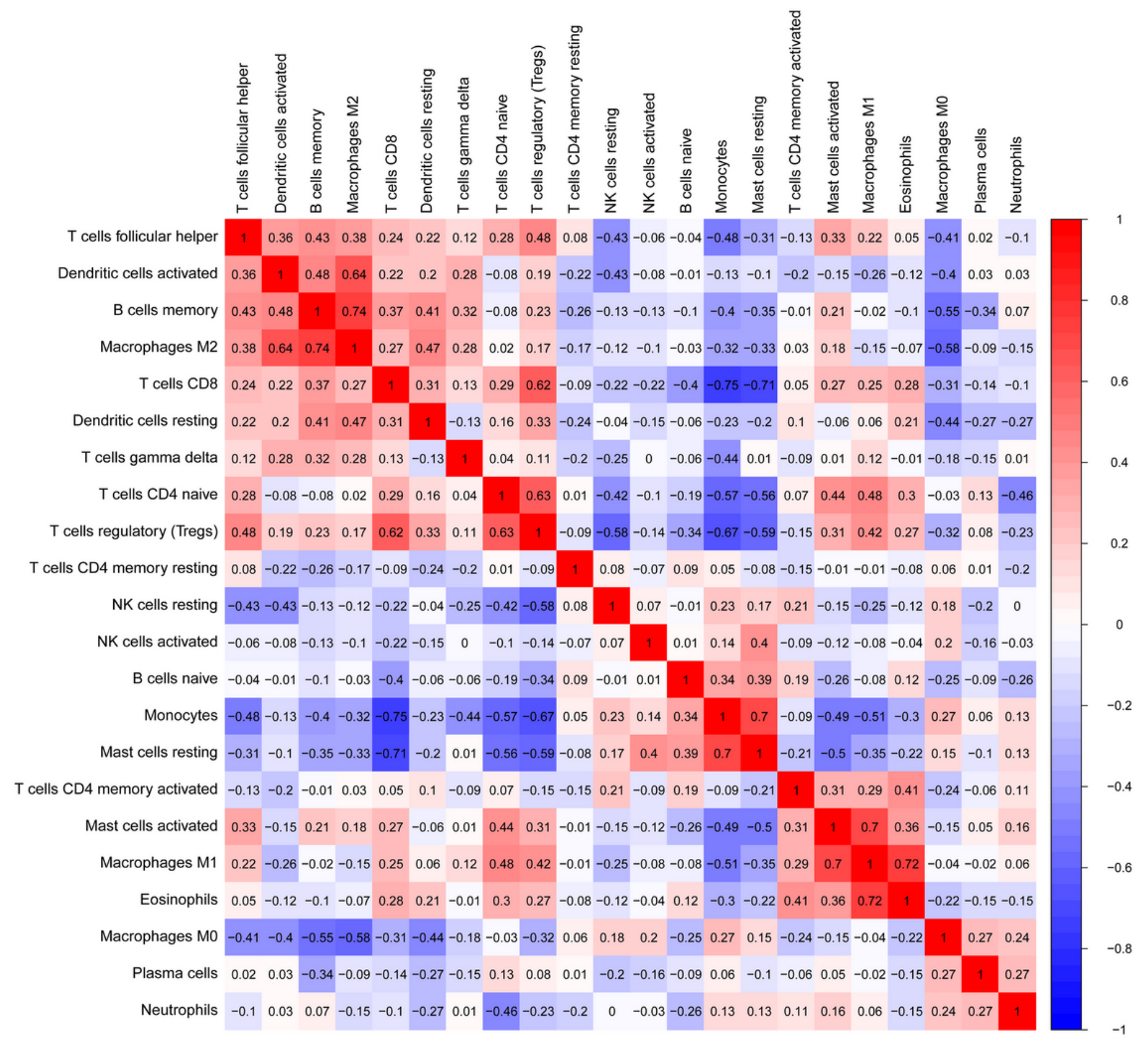

\section{Figure 2}

Correlation matrix of the degree of immune cell infiltration in FA samples. Red = a positive correlation; blue $=$ a negative correlation . 


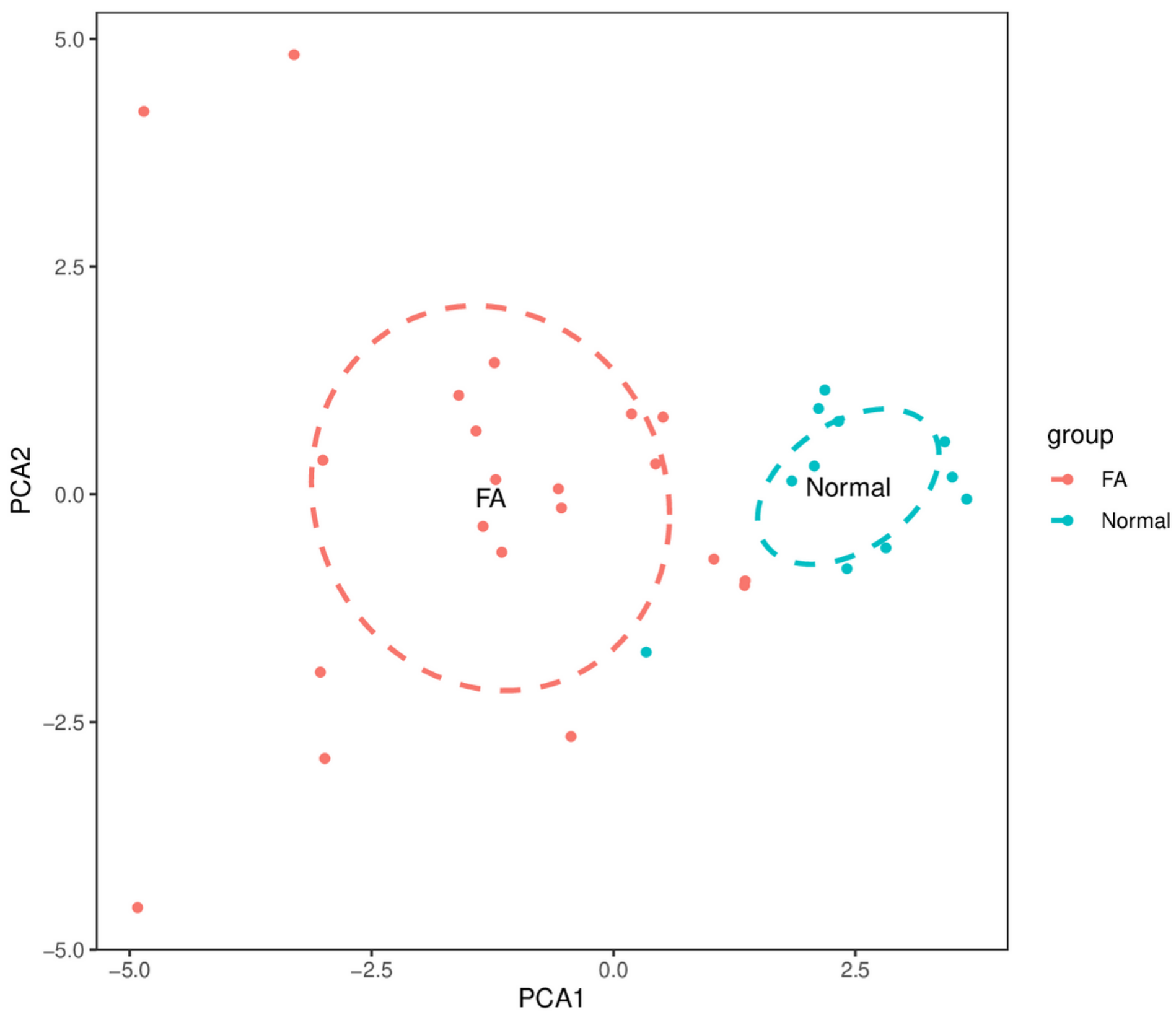

Figure 3

Principal component analysis $($ PCA $)$. Red points $=$ FA samples; blue points $=$ normal samples . 
A

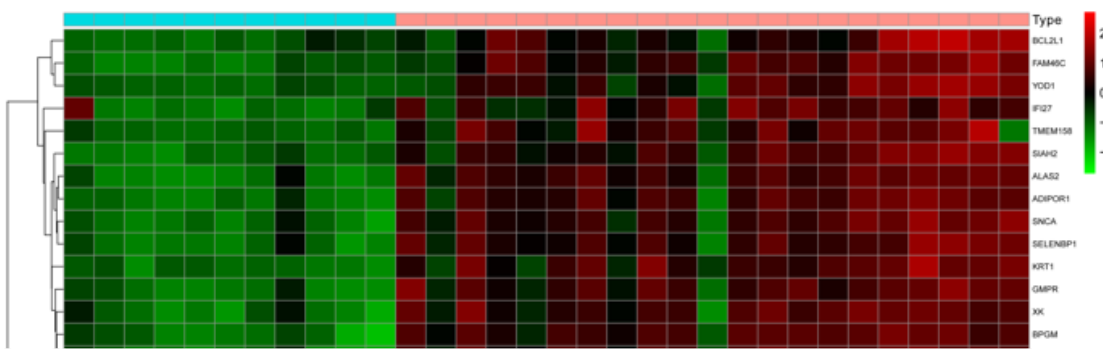

B

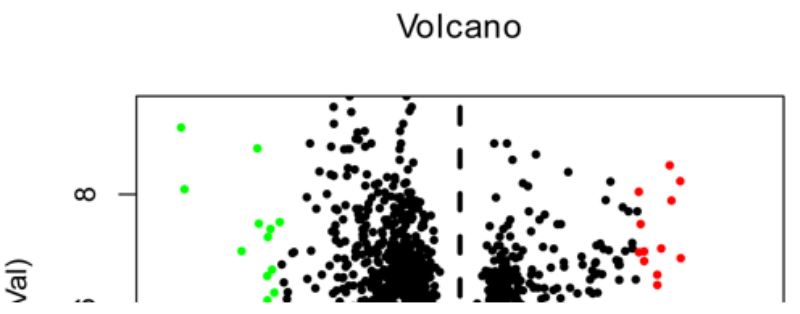

\section{Figure 4}

Identification of DEGs. (A) Heatmap of 37 upregulated and downregulated DEGs.

Red = highly expressed DEGs; green = lowly expressed DEGs. Explanation of "type": blue = normal tissue; red = FA tissue. (B) Volcano plot of the differentially expressed genes between FA and normal tissues $\bigotimes$ the genes represented by the green and red dots were statistically different. 


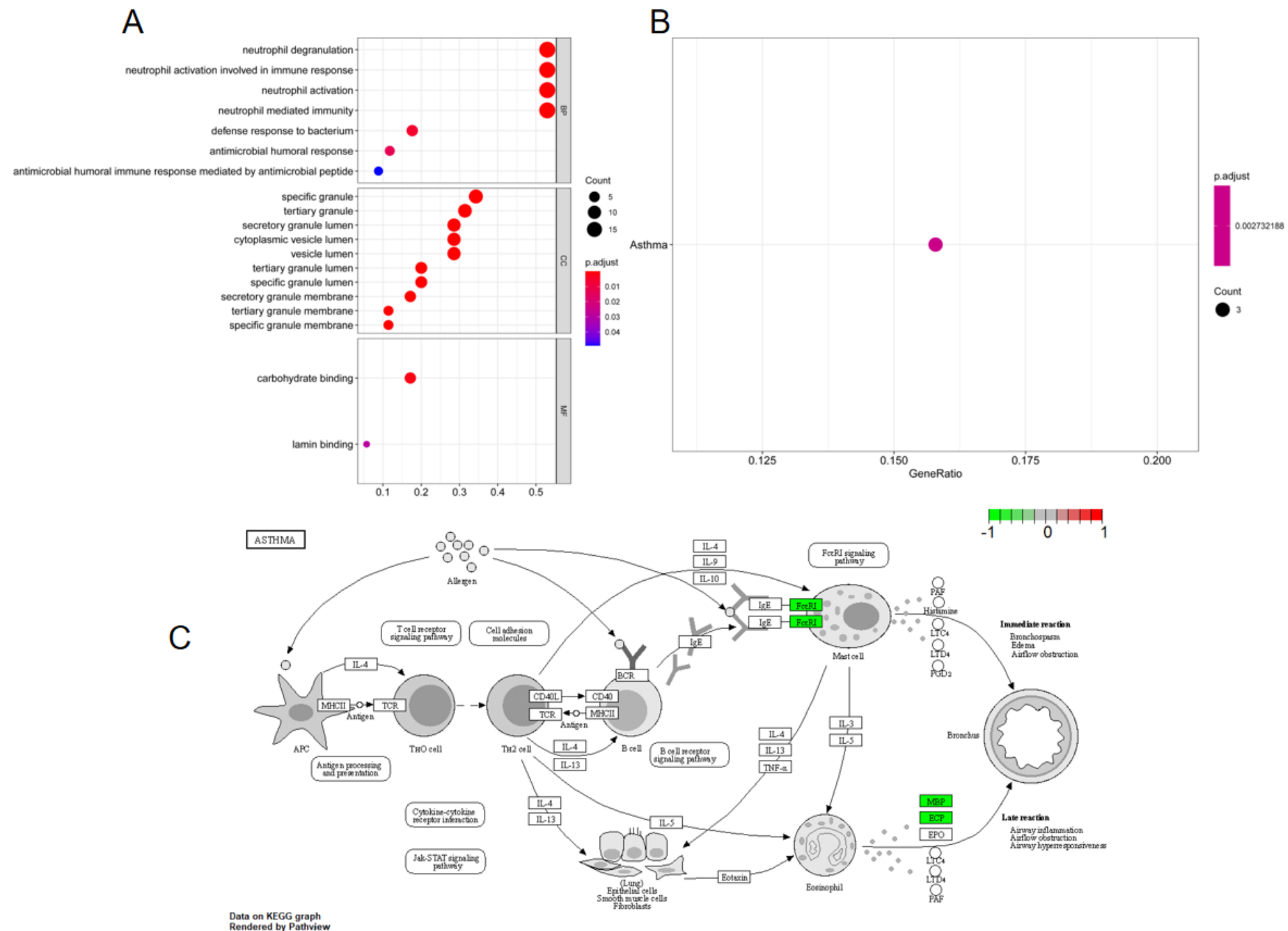

Figure 5

GO and KEGG pathway enrichment analysis of DEGs in GSE16334. (A) GO enrichment analysis. (B) KEGG pathway enrichment analysis. (C) KEGG pathway map for FA. 